\title{
Interactive comment on "Uncertainties in projections of the Baltic Sea ecosystem driven by an ensemble of global climate models" by Sofia Saraiva et al.
}

\section{Sofia Saraiva et al.}

sofia.maretec@tecnico.ulisboa.pt

Received and published: 4 June 2018

[12pt]article graphics

Answers to the comments submitted by anonymous Referee \#1 concerning the manuscript entitled "Uncertainties in projections of the Baltic Sea ecosystem driven by an ensemble of global climate models" by Sofia Saraiva et al. 
of specific terms used in climate modelling rather than language issues. We rephrased large parts of the text and added a table that explains acronyms (Tab. 1). We hope that the manuscript is now better readable for the reviewer and the readership of ESD. The answer to specific comments are addressed bellow and a new version of the manuscript can be found as a supplement to this comment.

ESDD

Interactive

comment

This article attempted to explore the uncertainties of biogeochemistry component, such as primary production, nitrogen fixation, and hypoxia area by using numerical experiments within Baltic Sea region. These uncertainties then discussed by various numerical scenarios among the change of nutrient loads, global model deficiencies and future greenhouse gas scenarios. Although the context looks well fit the ESD journal, the writing skills of authors made a hard time to me to read through the entire paper. In such case, I would like to suggest giving the rejection or requesting the major revisions in the way of the writing first. Here are some examples I would like to give.

1. The abstract mentioned a jargon "transient simulations", which also exist within the introduction section; however, the authors didn't declare/define what it is.

Answer: The concept of transient simulations is common within the climate modelling community in contrast to the time slice approach. Some time ago, for several reasons, models were not able to perform continuous simulations of 100 years. Thus, the modelling approaches were based on time slices: the model would run for about 30 years in the present climate and for another 30 years in the future. That approach was the possible one, but it had several faults (that we will not explore now but for example the initialization of the future period). With the increase in computational resources this limitation does not exist anymore, and we were able today to perform continuous, transient simulation. To avoid confusion, we omitted 'transient'.

Printer-friendly version

Discussion paper 
2. Line 22 on page 2 addressed 'approximately 18 years ago' to begin the sentence, but the sentence or the sentences nearby did not mentioned the reference year of '18 years ago'. As a reader, it is hard to capture what the period of '18 years ago' is meaning? Is it the 18 year ago of 2018, 2017, 2006 or what?

Answer: We agree and changed the text accordingly.

3. The authors keep using "GCM" or "GCMs", but didn't mentioned/defined what GCMs are. For example, line 25 on page 2, "The first scenario simulations were based on only one GCM and one greenhouse gas emission scenario, .... to future climates utilizing mini-ensembles consisting of two GCMs and two emission scenarios .... even used three GCMs and three emission scenarios." This is hard to understand what "GCMs" and what "scenarios" the previous studies worked. It is also difficult to realize the thoughts of authors why they want to mention those of previous works, even though reader can "guess" the authors want to address the uncertainties issue from the usage of GCMs use.

Answer: GCM stands for General Circulation model and was defined in the abstract and in the introduction when GCM is mentioned for the first time.

4. The name of section "Methods" should use "data and methodologies" instead, because you are only just saying what methods you will use but also the data you apply.

Answer: We changed the title of the sub-section.

5. Again, it is confused by the abbreviations authors used. For example, line 17 on page 4 saying "The Baltic Sea model was forced by (1) atmospheric surface fields from a regional coupled atmosphere-ocean climate model (RCM) driven by lateral boundary conditions from GCMs and by (2) runoff and (3) nutrient loads
ESDD

Interactive

comment
Printer-friendly version

Discussion paper

\section{3}


from a regional hydrological model also forced by regionalized atmospheric data from the same GCMs. The RCM is RCA4-NEMO applied to the EURO-CORDEX ESDD domain with an interactively coupled Baltic Sea and North Sea." Is it necessary to give the abbreviation "RCM" to use in the sentence? Is it possible to just use RCA4-NEMO to instead? What is RAC4-NEMA? What is EURO-CORDEX domain? If RCA4-NEMO is coupled model, why you only briefly explain the RCA4 but no description for the ocean component? Also, the description is too shallow to understand what it is.

Answer: We believe that it is important to specify that our methodology uses results from a coupled atmosphere-ocean RCM model (regional climate model) because that is in fact a distinction from other studies. We realize that the high number of abbreviations can be difficult to read but most of them (e.g. EURO-CORDEX or NEMO model) are very common within the climate science community. Also, considering that the manuscript is already long, we made the option to describe briefly the used models, though providing references for a more detailed description. Nevertheless, the subsection on 'Regional climate data sets' of the manuscript was reformulated and expanded to clarify the description of the different models.

6. There is no connection between sentences and sentences. For example, in the line 21 on page 4, "RAC4 has a 0.22-degree spherical rotated latitude/longitude grid with 40 vertical levels. The hydrological model is E-HYPE, a process-based multi model applied for Europe. The runoff from each river was corrected by a factor : : :: : : From these few sentences, it is understood that there is an atmospheric component called RCA4, but there is no clue saying that the E-HYPE is the ocean component of the coupled model.

Answer: We hope that the rephrased sentences are now clearer in the manuscript.

Interactive

comment

Printer-friendly version

Discussion paper 
7. Line 24 on page 4. "The runoff from each river was corrected by a factor that corresponds to the ratio : : : This approach has been previously applied for regional climate simulations of the Baltic Sea." Authors did not explain it clearly why they want to use this ratio to correct the river runoff. In addition, the sentence I addressed above didn't have the connection with the sentence below "here, improved versions of the regional and global climate models and the results of scenario simulations from the latest IPCC assessment report were used." It is hard to understand the authors want to speak out again.

Answer: Following the previous remark, the sentences are rephrased. The runoff from the E-HYPE model was corrected so that the model results match the observations in the historical period. This decision was made after realizing that the bias in the E-HYPE model could affect significantly the hydrodynamic results.

8. Line 30 on page 4. "RCP 4.5 and 8.5 are medium and high-end scenarios, respectively." As a reader, it is no sense to know what are RCP4.5 and RCP8.5, what those scenarios look like. This is the duties of authors to declare their words clearly rather than the readers guess the authors' thoughts.

Answer: RCP 4.5 and RCP 8.5 are common abbreviations for the two most relevant Representative Concentration Pathways adopted by the IPCC and its detail description would be out of scope of the manuscript. For that reason, we invite the reader to follow the references provided in the text. Nevertheless, the text was expanded.

9. Line 1 on page 5. "The selection of these GCMs follows the approach presented by Wilcke and Barring (2016)". Again, what is the "approach" you are saying it.

Interactive

comment

Printer-friendly version

Discussion paper 
You need to explain it.

Answer: For clarification we rephrased the text. The approach by Wilcke and Bärring (2016) is now briefly described: 'The selected models agree with the results obtained by Wilke and Bärring (2016) for the regional climate systems of the North Sea and Baltic Sea, which used hierarchical clustering methods to select an optimum subset of models, from the entire ensemble, to estimate uncertainties inherent in an ensemble with a minimum number of simulations.'

10. Line 4 on page 5. "The four driving GCMs of this study were selected from the distinct clusters identified by Wilcke and Bärring (2016)." Is this redundant sentence comparing with line 1 on page 5 ?

Answer: Yes, the reviewer's comment is correct. The sentence was redundant and it was changed.

11. Line 5 on page 5. What is "necessary/unnecessary lateral boundary data"? Meanwhile it is little bit confused about the selected GCMs came from. It is "Wilcke and Barring" or "SMHI".

Answer: The sentence was rephrased.

12. Line 7 to Line 11 on page 5. 1. High quality reanalysis dataset EURO4M. What is the definition of "high quality"? Is ERA40 low quality reanalysis? Than why do not compare with ERA-Interim or other ECMWF products? Or what do not you to compare with satellite observed winds? 2. Where is the correct factor 1.6 coming from? Why is 1.6? Why is not 2.0, 3.0, or 4.0? You didn't mention it.

Answer: The reviewer's comment is correct. 'high quality' term was removed from the text. The factor of 1.6 comes from comparing the winds at selected positions over sea in respective dataset. When the wind speeds are plotted
ESDD

Interactive

comment
Printer-friendly version

Discussion paper 
against each other (sorted individually) they form a rather straight line with a knee at $10 \mathrm{~m} / \mathrm{s}$ and a slope above the knee that indicates a factor of 1.6 wrong. The factor 1.6 comes from manual fitting of a line. We can say that is not 2.0 but we can not say if it should be 1.61 instead. We have extended the text to explain this but without going into any details.

13. Line 12 to Line 18 on page 5. 1. You were mentioned the wind correction of RCA4-NEMO output, and then jump to an example. Normally, this example should be the example of your wind correction; however, you are addressing the "air temperature" and "total runoff" as examples. It is hard to see the connection between the sentences.

Answer: The reviewer is correct. The link between the sentences was perhaps not the best and the wording has been improved in the revised version.

14. Meanwhile, what air temperature you are using? On the surface layer? On the $850 \mathrm{mb}$, or on $200 \mathrm{mb}$ ?

Answer: We analysed the $2 \mathrm{~m}$ air temperature which is a diagnostic output variable of atmospheric models. This is added to the text.

15. Since the previous description did not clearly describe the RCP 4.5 and RCP 8.5 , it is hard for readers to understand what "present" and "future" climates in your figures.

Answer: RCP 4.5 and RCP 8.5 are now clearly described in the manuscript as the Representative Concentration Pathways adopted by the IPCC in the latest assessment report.

16. When you use legend for your figures, you want to keep consistency between C7
ESDD

Interactive

comment

Printer-friendly version

Discussion paper

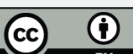


your figures and legends. You are using line with square in the legend, then should use line with square in the plots.

ESDD

Answer: We changed the text accordingly.

17. You are addressing the annual men for both air temperature and runoff. But you can only see the runoff annual mean plots existed, but no for air temperature. Where is it? Why do not you to show it?

Answer: We followed the suggestion by the reviewer and added the information about spatially averaged air temperature changes in RCP 4.5 and RCP 8.5 to the text. As we do not have observations we have not displayed the two numbers as bar plot.

18. Subsection of nutrient load scenarios. It is understood that there is three nutrient loads scenarios are applied in your study, but it is hard to understand why you want to pick up this three scenarios. It is better to describe the purposes and differences between each nutrient scenario clearly.

Answer: Changes were made in the description of the three scenarios that span a range of plausible future socio-economic conditions from the most optimistic to the worst scenario. The definition of the scenarios it's in fact quite strait forward: (1) one scenario that reflects the most optimistic possibility: the application of the BSAP; (2) the reference, meaning a scenario where nothing from the socio-economic point of view is changed (cities location, land use, industry) and the changes are only driven by climate changes; and the worst scenario were we assume not only climate change but also the worst socio-economical development, meaning the one with absolutely no care about the ecological impacts of socio-economic growth. We agree with the reviewer, that the definition of these scenarios becomes clearer by showing the differences between them. This is the reason why Fig. 4 is presented.

Interactive

comment

Printer-friendly version

Discussion paper

C8 
19. Line 34 on page 5 and Line 1 on page 6 . "are multiplied by a factor that summarizes the impact of a worst case socio-economic development on current nutrient loads. The main assumptions and description of this impact factor can be found in (Zandersen et al., in prep)." Here is the thing. You didn't explain why you want to multiple a factor. You didn't explain how did you obtain this factor. You didn't address the assumptions and description of this impact factor but addressing a non-published article. How does the reader know what/why/how you are doing this?

Answer: Changes were made in the manuscript to clarify this topic. However, the methodology to estimate the socio-economic impact factor is not detailed described in this manuscript, as it was not performed by the authors and its complexity deserves the reading of the referenced paper. Nevertheless, we have changed the manuscript to include some more details on what the impact factor is, referring that it takes into account the changes induced in the nutrient loads and atmosphere deposition by the adoption of each SSP (shared socio-economic pathways) in the Baltic Sea region.

20. Line 3 on page 6. "as well. In all three scenarios, nutrient loads : : :." The sentence and the previous sentence are not talking about the exactly the same thing. It is necessary to give a line break here.

Answer: A line break was added.

21. After read through the entire method section, It is difficult to understand that the authors are running regional coupled model - RCA4-NEMO or running the Baltic Sea model or running the four GCMs (MPI-ESM-LR, EC-EARTH,

Interactive comment
Printer-friendly version

Discussion paper 
IPSL-CM5a-MR, and HadGEM2-ES) to conduct their research.

Answer: We have used results from the regional climate model RCA4-NEMO

ESDD and results from the land surface model E-HYPE that both were run using four GCMs and two greenhouse emission scenarios to force a Baltic Sea model. We have revised the text to explain the methodology better.

Interactive

comment 